\title{
Bäcklund transformations for high-order constrained flows of the AKNS hierarchy: canonicity and spectrality property
}

\author{
Yunbo Zeng† Huihui Daił Jinping Song* \\ $\dagger$ Department of Mathematical Sciences, Tsinghua University, Beijing 100084, China \\ $\ddagger$ Department of Mathematics, City University of Hong Kong, Kowloon, Hong Kong, China \\ * Department of Mathematics, Henan University, Kaifeng 475001, Henan, China
}

\begin{abstract}
New infinite number of one- and two-point Bäcklund transformations (BTs) with explicit expressions are constructed for the high-order constrained flows of the AKNS hierarchy. It is shown that these BTs are canonical transformations including Bäcklund parameter $\eta$ and a spectrality property holds with respect to $\eta$ and the 'conjugated' variable $\mu$ for which the point $(\eta, \mu)$ belongs to the spectral curve. Also the formulas of m-times repeated Darboux transformations for the high-order constrained flows of the AKNS hierarchy are presented.
\end{abstract}

\section{Introduction}

Bäcklund transformations (BTs) are an important aspect of the theory of integrable systems [1, 2]. It is well-known that the BT's for soliton equations are canonical transformations ( see, for emaple, [3, 田, 5]). More recently there has been much interest in the property of BTs for finite-dimensional integrable Hamiltonian systems [6, 7, 8, 9, 10]. These BTs are defined as symplectic integrable maps which can be described explicitly and can be viewed as time discretization of particular flows of Liouville integrable systems. They are canonical transformations including Bäcklund parameter $\eta$. For these BTs a spectrality property holds with respect to $\eta$ and the 'conjugated' variable $\mu$ and the point $(\eta, \mu)$ or $(\eta, f(\mu))$ for some function $f(\mu)$ lies on the spectral curve. An important application of the spectrality property of BTs is that to the problem of separation 
of variables. In fact, the sequence of Bäcklund parameters $\eta_{j}$ together with the conjugate variables $\mu_{j}$ constitute the separation variables for the finite-dimensional integrable Hamiltonian systems [6].

We proceed to develop the ideas with some new BTs and study the problem of constructing one- and two-point Bäcklund transformation for the high-order constrained flows of the soliton hierarchy [11, 12, 13]. The Lax representation for the high-order constrained flows can always be deduced from the adjoint representation for the soliton hierarchy [14, 15]. Then, based on the results of Darboux transformations (DTs) for soliton hierarchy [2, 16, 17, 18, 20], we can find the DTs for the high-order constrained flows. By using the Lax representation, these DTs give rise to explicit one- and two-point BTs including one and two Bäcklund parameters $\eta_{i}$, respectively. We show these BTs to be canonical transformations by presenting their generating functions. Then we show that these BTs possess the spectrality property with respect to $\eta$ and conjugate variable $\mu$ and the pairs $\left(\eta_{i}, \mu_{i}\right)$ belong to the spectral curve, namely satisfy the separation equations. Few example of this kind of BTs were presented in [6, 7, 8, 9, 10]. This paper presents a way to find infinite number of BTs with the property described in [6, 7, 8, 9, 10 by means of DTs for the high-order constrained flows of soliton hierarchy. We will use the high-order constrained flows of the AKNS hierarchy to illustrate the ideas.

In section 2, we briefly describe the high-order constrained flows of the AKNS hierarchy. In section 3 we first present three kinds of DTs for the constrained flows of the AKNS hierarchy. Then we find infinite number of new one-point and two-point BTs from the first and third kind of DTs, respectively, and show them to be canonical transformations and possessing the spectrality property by using the first three high-order constrained flows as model in the section 3 and 4, respectively. Finally, the formula for m-times repeated DTs for the constrained flows are presented in section 5 .

\section{High-order constrained flows of the AKNS hierar- chy}

Let us briefly describe the high-order constrained flows of AKNS hierarchy. Consider the AKNS spectral problem [21]

$$
\psi_{x}=U(u, \lambda) \psi \equiv\left(\begin{array}{cc}
-\lambda & q \\
r & \lambda
\end{array}\right) \psi, \quad \psi=\left(\begin{array}{c}
\psi_{1} \\
\psi_{2}
\end{array}\right), \quad u=\left(\begin{array}{c}
q \\
r
\end{array}\right),
$$


and the evolution of $\psi$

$$
\psi_{t_{n}}=V^{(n)}(u, \lambda) \psi=\sum_{i=0}^{n}\left(\begin{array}{cc}
a_{i} & b_{i} \\
c_{i} & -a_{i}
\end{array}\right) \lambda^{n-i} \psi
$$

where

$$
\begin{gathered}
a_{0}=-1, \quad b_{0}=c_{0}=a_{1}=0, \quad b_{1}=q, \quad c_{1}=r \\
a_{2}=\frac{1}{2} q r, \quad b_{2}=-\frac{1}{2} q_{x}, \quad c_{2}=\frac{1}{2} r_{x}, \ldots,
\end{gathered}
$$

and in general

$$
\begin{gathered}
\left(\begin{array}{c}
c_{m+1} \\
b_{m+1}
\end{array}\right)=L\left(\begin{array}{c}
c_{m} \\
b_{m}
\end{array}\right)=L^{m}\left(\begin{array}{l}
r \\
q
\end{array}\right), \quad a_{m, x}=q c_{m}-r b_{m}, \\
L=\frac{1}{2}\left(\begin{array}{cc}
D-2 r D^{-1} q & 2 r D^{-1} r \\
-2 q D^{-1} q & -D+2 q D^{-1} r
\end{array}\right), \quad D=\frac{\partial}{\partial x}, \quad D D^{-1}=D^{-1} D=1 .
\end{gathered}
$$

Then the compatibility condition of Eqs. (2.1) and (2.2) gives rise to the AKNS hierarchy 21]

$$
u_{t_{n}}=\left(\begin{array}{c}
q \\
r
\end{array}\right)_{t_{n}}=J\left(\begin{array}{l}
c_{n+1} \\
b_{n+1}
\end{array}\right)=J \frac{\delta H_{n+1}}{\delta u}, \quad n=0,1, \cdots
$$

where

$$
H_{n}=\frac{2 a_{n+1}}{n}, \quad J=\left(\begin{array}{cc}
0 & -2 \\
2 & 0
\end{array}\right)
$$

We have

$$
\frac{\delta \lambda}{\delta q}=\psi_{2}^{2}, \quad \frac{\delta \lambda}{\delta r}=-\psi_{1}^{2}
$$

The high-order constrained flows of the AKNS hierarchy consist of the equations obtained from the spectral problem (2.1) for $N$ distinct $\lambda_{j}$ and the restriction of the variational derivatives for conserved quantities $H_{n}$ and $\lambda_{j}$ [11, 15]

$$
\begin{gathered}
\Phi_{1, x}=-\Lambda \Phi_{1}+q \Phi_{2}, \quad \Phi_{2, x}=r \Phi+\Lambda \Phi_{2}, \\
\frac{\delta H_{n+1}}{\delta u}-\alpha \sum_{j=1}^{N} \frac{\delta \lambda_{j}}{\delta u}=\left(\begin{array}{c}
c_{n+1} \\
b_{n+1}
\end{array}\right)-\alpha\left(\begin{array}{c}
<\Phi_{2}, \Phi_{2}> \\
-<\Phi_{1}, \Phi_{1}>
\end{array}\right)=0,
\end{gathered}
$$


where we have used $\left(\phi_{1 j}, \phi_{2 j}\right)^{T}$ to denote the solution of $(2.1)$ with $\lambda=\lambda_{j}, j=1, \ldots, N$ and $\Phi_{i}=\left(\phi_{i 1}, \ldots, \phi_{i N}\right)^{T}, i=1,2, \Lambda=\operatorname{diag}\left(\lambda_{1}, \ldots, \lambda_{N}\right),<., .>$ denotes the inner product. The Lax representation for the constrained flow (2.4) is given by [14, 15]

$$
M_{x}^{(n)}=\left[U, M^{(n)}\right],
$$

with Lax matrix $M^{(n)}$

$$
\begin{gathered}
M^{(n)}\left(u, \Phi_{1}, \Phi_{2}, \lambda\right)=\left(\begin{array}{cc}
A^{(n)} & B^{(n)} \\
C^{(n)} & -A^{(n)}
\end{array}\right)=V^{(n)}+M_{0} \\
M_{0}=\alpha \sum_{j=1}^{N} \frac{1}{\lambda-\lambda_{j}}\left(\begin{array}{cc}
\phi_{1 j} \phi_{2 j} & -\phi_{1 j}{ }^{2} \\
\phi_{2 j}{ }^{2} & -\phi_{1 j} \phi_{2 j}
\end{array}\right),
\end{gathered}
$$

and the Lax pair for (2.4)

$$
\begin{gathered}
\psi_{x}=U(u, \lambda) \psi, \\
M^{(n)}\left(u, \Phi_{1}, \Phi_{2}, \lambda\right) \psi=\mu \psi .
\end{gathered}
$$

The spectral curve $\Gamma$,

$$
\Gamma: \quad \operatorname{det}\left(M^{(n)}\left(u, \Phi_{1}, \Phi_{2}, \lambda\right)-\mu\right)=0
$$

is

$$
\mu^{2}=\left(A^{(n)}\right)^{2}(\lambda)+B^{(n)}(\lambda) C^{(n)}(\lambda) .
$$

We present the first three high-order constrained flows as follows.

(1) For $n=0, \alpha=\frac{1}{2}$, (2.4b) gives an explicit constraint

$$
q=-\frac{1}{2}<\Phi_{1}, \Phi_{1}>, \quad r=\frac{1}{2}<\Phi_{2}, \Phi_{2}>.
$$

Then (2.4a) becomes a finite-dimensional integrable Hamiltonian system (FDIHS)

$$
\begin{gathered}
\Phi_{1, x}=\frac{\partial \widetilde{H}_{0}}{\partial \Phi_{2}}, \quad \Phi_{2, x}=-\frac{\partial \widetilde{H}_{0}}{\partial \Phi_{1}} \\
\widetilde{H}_{0}=-<\Lambda \Phi_{1}, \Phi_{2}>-\frac{1}{4}<\Phi_{1}, \Phi_{1}><\Phi_{2}, \Phi_{2}>
\end{gathered}
$$

with Lax matrix $M^{(0)}$

$$
A^{(0)}=-1+\frac{1}{2} \sum_{j=1}^{N} \frac{1}{\lambda-\lambda_{j}} \phi_{1 j} \phi_{2 j}, \quad B^{(0)}=-\frac{1}{2} \sum_{j=1}^{N} \frac{1}{\lambda-\lambda_{j}} \phi_{1 j}^{2},
$$




$$
C^{(0)}=\frac{1}{2} \sum_{j=1}^{N} \frac{1}{\lambda-\lambda_{j}} \phi_{2 j}^{2}
$$

The spectral curve $\Gamma$ is a hyperelliptic, genus $N-1$ curve

$$
\mu^{2}=1+\sum_{j=1}^{N} \frac{P_{j}}{\lambda-\lambda_{j}}
$$

with

$$
P_{j}=-\phi_{1 j} \phi_{2 j}+\frac{1}{2} \sum_{k \neq j} \frac{1}{\lambda_{j}-\lambda_{k}}\left(\phi_{1 j} \phi_{2 j} \phi_{1 k} \phi_{2 k}-\phi_{1 k}^{2} \phi_{2 j}^{2}\right), \quad j=1, \ldots, N .
$$

$P_{1}, \ldots, P_{N}$ are $N$ independent integrals of motion in involution for FDIHS (2.11).

(2) For $n=1, \alpha=-\frac{1}{4}$, (2.4) can be written as a FDIHS

$$
Q_{x}=\frac{\partial \widetilde{H}_{1}}{\partial P}, \quad P_{x}=-\frac{\partial \widetilde{H}_{1}}{\partial Q}
$$

with

$$
\begin{gathered}
\widetilde{H}_{1}=-<\Lambda \Phi_{1}, \Phi_{2}>-\frac{1}{2} r<\Phi_{1}, \Phi_{1}>+\frac{1}{2} q<\Phi_{2}, \Phi_{2}> \\
Q=\left(\phi_{11}, \ldots, \phi_{1 N}, q\right)^{T}, \quad P=\left(\phi_{21}, \ldots, \phi_{2 N}, r\right)^{T}
\end{gathered}
$$

and Lax matrix $M^{(1)}$

$$
\begin{gathered}
A^{(1)}=-\lambda-\frac{1}{4} \sum_{j=1}^{N} \frac{1}{\lambda-\lambda_{j}} \phi_{1 j} \phi_{2 j}, \quad B^{(1)}=q+\frac{1}{4} \sum_{j=1}^{N} \frac{1}{\lambda-\lambda_{j}} \phi_{1 j}^{2}, \\
C^{(1)}=r-\frac{1}{4} \sum_{j=1}^{N} \frac{1}{\lambda-\lambda_{j}} \phi_{2 j}^{2} .
\end{gathered}
$$

The spectral curve $\Gamma$ is

$$
\mu^{2}=\lambda^{2}+P_{0}+\sum_{j=1}^{N} \frac{P_{j}}{\lambda-\lambda_{j}}
$$

with

$$
\begin{gathered}
P_{0}=\frac{1}{2}<\Phi_{1} \Phi_{2}>+q r \\
P_{j}=\frac{1}{4}\left(2 \lambda_{j} \phi_{1 j} \phi_{2 j}+r \phi_{1 j}^{2}-q \phi_{2 j}^{2}\right)+\frac{1}{8} \sum_{k \neq j} \frac{1}{\lambda_{j}-\lambda_{k}}\left(\phi_{1 j} \phi_{2 j} \phi_{1 k} \phi_{2 k}-\phi_{1 k}^{2} \phi_{2 j}^{2}\right) .
\end{gathered}
$$

$P_{0}, \ldots, P_{N}$ are $N+1$ independent integrals of motion in involution for FDIHS (2.14). 
(3) For $n=2, \alpha=\frac{1}{2}$, by introducing the following Jacobi-Ostrogradsky coordinates

$$
\begin{gathered}
Q=\left(\phi_{11}, \ldots, \phi_{1 N}, q_{1}, q_{2}\right)^{T}, \quad P=\left(\phi_{21}, \ldots, \phi_{2 N}, r_{1}, r_{2}\right)^{T}, \\
q_{1}=q, \quad q_{2}=r, \quad p_{1}=-\frac{1}{4} r_{x}, \quad p_{2}=-\frac{1}{4} q_{x},
\end{gathered}
$$

(2.4) can be transformed into a FDIHS

$$
Q_{x}=\frac{\partial \widetilde{H}_{2}}{\partial P}, \quad P_{x}=-\frac{\partial \widetilde{H}_{2}}{\partial Q},
$$

with

$$
\widetilde{H}_{2}=-<\Lambda \Phi_{1}, \Phi_{2}>-\frac{1}{2} q_{2}<\Phi_{1}, \Phi_{1}>+\frac{1}{2} q_{1}<\Phi_{2}, \Phi_{2}>+\frac{1}{4} q_{1}^{2} q_{2}^{2}-4 p_{1} p_{2},
$$

and Lax matrix $M^{(2)}$

$$
\begin{gathered}
A^{(2)}=-\lambda^{2}+\frac{1}{2} q_{1} q_{2}+\frac{1}{2} \sum_{j=1}^{N} \frac{1}{\lambda-\lambda_{j}} \phi_{1 j} \phi_{2 j}, \quad B^{(2)}=q_{1} \lambda+2 p_{2}-\frac{1}{2} \sum_{j=1}^{N} \frac{1}{\lambda-\lambda_{j}} \phi_{1 j}^{2}, \\
C^{(2)}=q_{2} \lambda-2 p_{1}+\frac{1}{2} \sum_{j=1}^{N} \frac{1}{\lambda-\lambda_{j}} \phi_{2 j}^{2}
\end{gathered}
$$

The spectral curve $\Gamma$ is

$$
\mu^{2}=\lambda^{4}+P_{0} \lambda+P_{N+1}+\sum_{j=1}^{N} \frac{P_{j}}{\lambda-\lambda_{j}},
$$

with

$$
\begin{gathered}
P_{0}=-<\Phi_{1} \Phi_{2}>-2 q_{1} p_{1}+2 p_{2} q_{2}, \quad P_{N+1}=\widetilde{H}_{2}, \\
P_{j}=\frac{1}{2}\left(-2 \lambda_{j}^{2} \phi_{1 j} \phi_{2 j}-\lambda_{j} q_{2} \phi_{1 j}^{2}+\lambda_{j} q_{1} \phi_{2 j}^{2}+q_{1} q_{2} \phi_{1 j} \phi_{2 j}\right)+p_{1} \phi_{1 j}^{2}+p_{2} \phi_{2 j}^{2} \\
+\frac{1}{2} \sum_{k \neq j} \frac{1}{\lambda_{j}-\lambda_{k}}\left(\phi_{1 j} \phi_{2 j} \phi_{1 k} \phi_{2 k}-\phi_{1 k}^{2} \phi_{2 j}^{2}\right), \quad j=1, \ldots, N .
\end{gathered}
$$

$P_{0}, \ldots, P_{N+1}$ are $N+2$ independent integrals of motion in involution for FDIHS (2.17). 


\section{One-point BTs for high-order constrained flows of the AKNS hierarchy}

We first briefly review the Darboux transformations (DTs) for the AKNS hierarchy. Suppose that a gauge transformation

$$
\bar{\psi}=T \psi
$$

transforms (2.1) and (2.2) into

$$
\begin{gathered}
\bar{\psi}_{x}=\bar{U}(\bar{u}, \lambda) \bar{\psi} \\
\bar{\psi}_{t_{n}}=\bar{V}^{(n)}(\bar{u}, \lambda) \bar{\psi}
\end{gathered}
$$

Let $\psi\left(x, \eta_{i}\right)$ be a solution of (2.1) and (2.2) with $\lambda=\eta_{i}, i=1,2, \eta_{i} \neq \lambda_{j}$. It is known [2, 16, 17, 18 that there are the following three kinds of the DTs for the AKNS hierarchy.

(1) The first DT for the AKNS hierarchy is given by

$$
T_{1}=\left(\begin{array}{cc}
\lambda-\eta_{1}+\frac{1}{2} q f_{1} & -\frac{1}{2} q \\
-f_{1} & 1
\end{array}\right), \quad f_{i}=\frac{\psi_{2}\left(x, \eta_{i}\right)}{\psi_{1}\left(x, \eta_{i}\right)}
$$

and

$$
\bar{q}=-\frac{1}{2} q_{x}-\eta_{1} q+\frac{1}{2} q^{2} f_{1}, \quad \bar{r}=2 f_{1}
$$

namely under the transformation (3.1) with (3.4) and (3.5), $\bar{U}$ and $\bar{V}^{(n)}$ are of the same form as $U$ and $V^{(n)}$ except for replacing $q$ and $r$ by $\bar{q}$ and $\bar{r}$. So (3.5) presents the relationship between two solutions $(q, r)$ and $(\bar{q}, \bar{r})$ of the equation (2.3).

(2) The second DT for the AKNS hierarchy is given by

$$
T_{2}=\left(\begin{array}{cc}
1 & -f_{2} \\
\frac{1}{2} r & \lambda-\eta_{2}-\frac{1}{2} r f_{2}
\end{array}\right)
$$

and

$$
\bar{q}=-2 f_{2}, \quad \bar{r}=\frac{1}{2} r_{x}-\eta_{2} r-\frac{1}{2} r^{2} f_{2} .
$$

(3) The third DT for the AKNS hierarchy is given by

$$
T_{3}=\left(\begin{array}{cc}
\lambda-\eta_{1}+m_{2} & -m_{1} \\
m_{3} & \lambda-\eta_{2}-m_{2}
\end{array}\right),
$$


and

$$
\bar{q}=q-2 m_{1}, \quad \bar{r}=r-2 m_{3},
$$

with

$$
\begin{array}{r}
m_{1}=\frac{\left(\eta_{2}-\eta_{1}\right) \psi_{1}\left(\eta_{1}\right) \psi_{1}\left(\eta_{2}\right)}{\triangle}, \quad m_{2}=\frac{\left(\eta_{2}-\eta_{1}\right) \psi_{1}\left(\eta_{2}\right) \psi_{2}\left(\eta_{1}\right)}{\triangle}, \\
m_{3}=\frac{\left(\eta_{2}-\eta_{1}\right) \psi_{2}\left(\eta_{1}\right) \psi_{2}\left(\eta_{2}\right)}{\triangle}, \quad \triangle=\psi_{1}\left(\eta_{1}\right) \psi_{2}\left(\eta_{2}\right)-\psi_{2}\left(\eta_{1}\right) \psi_{1}\left(\eta_{2}\right) .
\end{array}
$$

We now consider the DTs for high-order constrained flows (2.4). Suppose that the gauge transformation (3.1) and accordingly

$$
\left(\begin{array}{c}
\bar{\phi}_{1 j} \\
\bar{\phi}_{2 j}
\end{array}\right)=\beta_{j} T\left(\begin{array}{c}
\phi_{1 j} \\
\phi_{2 j}
\end{array}\right),
$$

transforms (2.7) and (2.8) into

$$
\begin{gathered}
\bar{\psi}_{x}=\bar{U}(\bar{u}, \lambda) \bar{\psi} \\
\bar{M}^{(n)}\left(\bar{u}, \bar{\Phi}_{1}, \bar{\Phi}_{2}, \lambda\right) \bar{\psi}=\mu \bar{\psi}
\end{gathered}
$$

where $\bar{U}$ and $\bar{M}^{(n)}$ satisfy

$$
\begin{gathered}
T_{x}=\bar{U}(\bar{u}, \lambda) T-T U(u, \lambda), \\
\bar{M}^{(n)}\left(\bar{u}, \bar{\Phi}_{1}, \bar{\Phi}_{2}, \lambda\right) T=T M^{(n)}\left(u, \Phi_{1}, \Phi_{2}, \lambda\right) .
\end{gathered}
$$

Motivated by the first DT for the AKNS hierarchy, let $\psi\left(x, \eta_{i}\right)$ be a solution of (2.7) and (2.8) with $\lambda=\eta_{i}, \mu=\mu_{i}, i=1,2, \eta_{i} \neq \lambda_{j}$. We find that the first DT for the constrained flows (2.4) consists of (3.1), (3.4), (3.5) and (3.11) with $\beta_{j}=\frac{1}{\sqrt{\lambda_{j}-\eta_{1}}}$, namely

$$
\begin{gathered}
\bar{\phi}_{1 j}=\sqrt{\lambda_{j}-\eta_{1}} \phi_{1 j}-\frac{1}{2 \sqrt{\lambda_{j}-\eta_{1}}} q\left(\phi_{2 j}-f_{1} \phi_{1 j}\right), \\
\bar{\phi}_{2 j}=\frac{1}{\sqrt{\lambda_{j}-\eta_{1}}}\left(\phi_{2 j}-f_{1} \phi_{1 j}\right), \quad j=1, \ldots, N .
\end{gathered}
$$

In fact, based on the results of the DTs for the AKNS hierarchy, it can be shown by a similar way in [19, 20] that under the transformation (3.1), (3.4), (3.5) and (3.16), $\bar{U}$ 
and $\bar{M}^{(n)}$ permit the same form as $U$ and $M^{(n)}$ except for replacing $q, r, \phi_{1 j}, \phi_{2 j}$ by $\bar{q}, \bar{r}$, $\bar{\phi}_{1 j}, \bar{\phi}_{2 j}$, namely, we have

$$
\begin{gathered}
T_{x}=U(\bar{u}, \lambda) T-T U(u, \lambda), \\
M^{(n)}\left(\bar{u}, \bar{\Phi}_{1}, \bar{\Phi}_{2}, \lambda\right) T=T M^{(n)}\left(u, \Phi_{1}, \Phi_{2}, \lambda\right) .
\end{gathered}
$$

The equalities (3.17) and (3.18) ensure that (2.7) and (2.8) are invarint under the transformation (3.1), (3.4), (3.5) and (3.16). This guarantees that the relationship between $q, r, \phi_{1 j}, \phi_{2 j}$ and $\bar{q}, \bar{r}, \bar{\phi}_{1 j}, \bar{\phi}_{2 j}$ obtained from (3.17) and (3.18) is just the one between two solutions of the constrained flows (2.4). In fact, (3.17) and (3.18) give rises to (3.5) and (3.16) which present the relationship between two solutions of the constrained flows (2.4).

It follows from (2.8)

$$
f_{i}=\frac{\mu_{i}-A^{(n)}\left(\eta_{i}\right)}{B^{(n)}\left(\eta_{i}\right)}=\frac{C^{(n)}\left(\eta_{i}\right)}{\mu_{i}+A^{(n)}\left(\eta_{i}\right)}, \quad i=1,2 .
$$

By substituting (3.19) into (3.5) and (3.16), we obtain infinite number $(n=0,1, \ldots)$ of the first explicit one-point BT $B_{\eta_{1}}$ for the constrained flows (2.4) as follows

$$
\begin{gathered}
\bar{q}=-\frac{1}{2} q_{x}-\eta_{1} q+\frac{1}{2} q^{2} \frac{\mu_{1}-A^{(n)}\left(\eta_{1}\right)}{B^{(n)}\left(\eta_{1}\right)}, \quad \bar{r}=2 \frac{\mu_{1}-A^{(n)}\left(\eta_{1}\right)}{B^{(n)}\left(\eta_{1}\right)}, \\
\bar{\phi}_{1 j}=\sqrt{\lambda_{j}-\eta_{1}} \phi_{1 j}-\frac{q}{2 \sqrt{\lambda_{j}-\eta_{1}}}\left(\phi_{2 j}-\frac{\mu_{1}-A^{(n)}\left(\eta_{1}\right)}{B^{(n)}\left(\eta_{1}\right)} \phi_{1 j}\right), \\
\bar{\phi}_{2 j}=\frac{1}{\sqrt{\lambda_{j}-\eta_{1}}}\left(\phi_{2 j}-\frac{\mu_{1}-A^{(n)}\left(\eta_{1}\right)}{B^{(n)}\left(\eta_{1}\right)} \phi_{1 j}\right) .
\end{gathered}
$$

It is found from (3.4) and (3.18)

$$
\begin{gathered}
\left(\lambda-\eta_{1}\right) \bar{A}^{(n)}(\lambda)=\left(\lambda-\eta_{1}+q f_{1}\right) A^{(n)}(\lambda)+f_{1}\left(\lambda-\eta_{1}+\frac{1}{2} q f_{1}\right) B^{(n)}(\lambda)-\frac{1}{2} q C^{(n)}(\lambda), \\
\left(\lambda-\eta_{1}\right) \bar{B}^{(n)}(\lambda)=q\left(\lambda-\eta_{1}+\frac{1}{2} q f_{1}\right) A^{(n)}(\lambda)+\left(\lambda-\eta_{1}+\frac{1}{2} q f_{1}\right)^{2} B^{(n)}(\lambda)-\frac{1}{4} q^{2} C^{(n)}(\lambda), \\
\left(\lambda-\eta_{1}\right) \bar{C}^{(n)}(\lambda)=-2 f_{1} A^{(n)}(\lambda)-f_{1}^{2} B^{(n)}(\lambda)+C^{(n)}(\lambda),
\end{gathered}
$$


which, as we mentioned above, present the relationship between two solutions of the constrained flows (2.4).

Using the first three constrained flows as model, we now show the BTs (3.20) to be canonical transformations by presenting their generating functions and check spectrality property with respect to the Bäcklund parameter $\eta$ and the 'conjugated' variable $\mu$ with the point $(\eta, \mu)$ belonging to the spectral curve (2.9).

(1) For the first constrained flow, the FDIHS (2.11), using (2.12) and comparing the coefficients of $\lambda^{0}$ in (3.210), one gets

$$
f_{1}=\frac{1}{4}<\bar{\Phi}_{2}, \bar{\Phi}_{2}>
$$

Then we have from (3.16)

$$
\begin{gathered}
\phi_{2 j}=\sqrt{\lambda_{j}-\eta_{1}} \bar{\phi}_{2 j}+\frac{1}{4}<\bar{\Phi}_{2}, \bar{\Phi}_{2}>\phi_{1 j}=\frac{\partial S^{(0)}}{\partial \phi_{1 j}}, \\
\bar{\phi}_{1 j}=\sqrt{\lambda_{j}-\eta_{1}} \phi_{1 j}+\frac{1}{4}<\Phi_{1}, \Phi_{1}>\bar{\phi}_{2 j}=\frac{\partial S^{(0)}}{\partial \bar{\phi}_{2 j}}
\end{gathered}
$$

where the generating function $S^{(0)}$ for the canonical transformation $(3.20)$ is given by

$$
S^{(0)}=\frac{1}{8}<\Phi_{1}, \Phi_{1}><\bar{\Phi}_{2}, \bar{\Phi}_{2}>+\sum_{j=1}^{N} \sqrt{\lambda_{j}-\eta_{1}} \phi_{1 j} \bar{\phi}_{2 j}-\eta_{1} .
$$

Furthermore, it is found from (2.12) and (3.16)

$$
\begin{gathered}
\frac{\partial S^{(0)}}{\partial \eta_{1}}=-1-\frac{1}{2} \sum_{j=1}^{N} \frac{1}{\sqrt{\lambda_{j}-\eta_{1}}} \phi_{1 j} \bar{\phi}_{2 j} \\
=-1-\frac{1}{2} \sum_{j=1}^{N} \frac{1}{\sqrt{\lambda_{j}-\eta_{1}}} \phi_{1 j} \frac{1}{\sqrt{\lambda_{j}-\eta_{1}}}\left[\phi_{2 j}-f_{1} \phi_{1 j}\right]=A^{(0)}\left(\eta_{1}\right)+f_{1} B^{(0)}\left(\eta_{1}\right)=\mu_{1},
\end{gathered}
$$

which implies that $\left(\eta_{1}, \mu_{1}\right)$ satisfies the spectrality property. Consider the composition $B_{\eta_{1} \ldots \eta_{N}}=B_{\eta_{1}} \circ \ldots \circ B_{\eta_{N}}$ of the Bäcklund transformation $B_{\eta_{i}}$. Then the corresponding generating function $S_{\eta_{1} \ldots \eta_{N}}^{(0)}$ becomes the generating function of the canonical transformation from $\left(\Phi_{1}, \Phi_{2}\right)$ to $(\eta, \mu)$ given by the equations

$$
\phi_{2 j}=\frac{\partial S_{\eta_{1} \ldots \eta_{N}}^{(0)}}{\partial \phi_{1 j}}, \quad \mu_{j}=\frac{\partial S_{\eta_{1} \ldots \eta_{N}}^{(0)}}{\partial \eta_{j}} .
$$


The points $\left(\eta_{i}, \mu_{i}\right)$ satisfy the separation equations given by the spectral curve (2.13)

$$
\mu_{i}^{2}=1+\sum_{j=1}^{N} \frac{P_{j}}{\eta_{i}-\lambda_{j}}, \quad i=1, \ldots, N .
$$

(2) For the second constrained flow, the FDIHS (2.14), using (2.15) and comparing the coefficients of $\lambda, \lambda^{0}$ in (3.21C) and coefficient of $\lambda$ in (3.21b), one gets $f_{1}=\frac{1}{2} \bar{r}$ and

$$
\begin{gathered}
r=-\frac{1}{4}<\bar{\Phi}_{2}, \bar{\Phi}_{2}>-\eta_{1} \bar{r}+\frac{1}{4} q \bar{r}^{2}=\frac{\partial S^{(1)}}{\partial q}, \\
\bar{q}=\frac{1}{4}<\Phi_{1}, \Phi_{1}>-\eta_{1} q+\frac{1}{4} q^{2} \bar{r}=\frac{\partial S^{(1)}}{\partial \bar{r}},
\end{gathered}
$$

then using (3.16)

$$
\begin{gathered}
\phi_{2 j}=\sqrt{\lambda_{j}-\eta_{1}} \bar{\phi}_{2 j}+\frac{1}{2} \bar{r} \phi_{1 j}=\frac{\partial S^{(1)}}{\partial \phi_{1 j}}, \\
\bar{\phi}_{1 j}=\sqrt{\lambda_{j}-\eta_{1}} \phi_{1 j}-\frac{1}{2} q \bar{\phi}_{2 j}=\frac{\partial S^{(1)}}{\partial \bar{\phi}_{2 j}},
\end{gathered}
$$

where the generating function $S^{(1)}$ for the canonical transformation (3.20) is given by

$$
S^{(1)}=\frac{1}{4} \bar{r}<\Phi_{1}, \Phi_{1}>-\frac{1}{4} q<\bar{\Phi}_{2}, \bar{\Phi}_{2}>-\eta_{1} q \bar{r}+\frac{1}{8} q^{2} \bar{r}^{2}+\sum_{j=1}^{N} \sqrt{\lambda_{j}-\eta_{1}} \phi_{1 j} \bar{\phi}_{2 j}+\eta_{1}^{2} .
$$

Furthermore, it is easy to check the spectrality property by means of (3.16) and (2.15)

$$
\begin{gathered}
\frac{\partial S^{(1)}}{\partial \eta_{1}}=-\frac{1}{2} \sum_{j=1}^{N} \frac{1}{\sqrt{\lambda_{j}-\eta_{1}}} \phi_{1 j} \bar{\phi}_{2 j}-q \bar{r}+2 \eta_{1} \\
=-2\left[A^{(1)}\left(\eta_{1}\right)+f_{1} B^{(1)}\left(\eta_{1}\right)\right]=-2 \mu_{1} .
\end{gathered}
$$

The point $\left(\eta_{1}, \mu_{1}\right)$ satisfies the separation equation given by the spectral curve (2.16)

$$
\mu_{1}^{2}=\eta_{1}^{2}+P_{0}+\sum_{j=1}^{N} \frac{P_{j}}{\eta_{1}-\lambda_{j}}
$$


(3) For the third constrained flow, the FDIHS (2.17), using (2.18), (3.16) and (3.21) in the same way as for (3.28), one gets $f_{1}=\frac{1}{2} \bar{q}_{2}$ and

$$
\begin{gathered}
\phi_{2 j}=\sqrt{\lambda_{j}-\eta_{1} \bar{\phi}_{2 j}+\frac{1}{2} \bar{q}_{2} \phi_{1 j}=\frac{\partial S^{(2)}}{\partial \phi_{1 j}},} \\
\bar{\phi}_{1 j}=\sqrt{\lambda_{j}-\eta_{1}} \phi_{1 j}-\frac{1}{2} q_{1} \bar{\phi}_{2 j}=\frac{\partial S^{(2)}}{\partial \bar{\phi}_{2 j}}, \\
\bar{q}_{1}=\frac{1}{4} q_{1}^{2} \bar{q}_{2}-\eta_{1} q_{1}+2 p_{2}=\frac{\partial S^{(2)}}{\partial \bar{p}_{1}} \\
q_{2}=\frac{1}{4} q_{1} \bar{q}_{2}^{2}-\eta_{1} \bar{q}_{2}-2 \bar{p}_{1}=-\frac{\partial S^{(2)}}{\partial p_{2}}
\end{gathered}
$$

then $p_{1}=-\frac{1}{4} q_{2 x}$ and $\bar{p}_{2}=-\frac{1}{4} \bar{q}_{1 x}$ lead

$$
\begin{gathered}
p_{1}=-\frac{1}{4}<\bar{\Phi}_{2}, \bar{\Phi}_{2}>-\eta_{1} \bar{p}_{1}+\frac{1}{2} q_{1} \bar{q}_{2} \bar{p}_{1}-\frac{1}{16} q_{1}^{2} \bar{q}_{2}^{3}+\frac{1}{4} q_{1} \bar{q}_{2}^{2} \eta_{1}-\frac{1}{4} \bar{q}_{2}^{2} p_{2}=\frac{\partial S^{(2)}}{\partial q_{1}} \\
\bar{p}_{2}=-\frac{1}{4}<\Phi_{1}, \Phi_{1}>-\eta_{1} p_{2}+\frac{1}{2} q_{1} \bar{q}_{2} p_{2}+\frac{1}{16} q_{1}^{3} \bar{q}_{2}^{2}-\frac{1}{4} q_{1}^{2} \bar{q}_{2} \eta_{1}-\frac{1}{4} q_{1}^{2} \bar{p}_{1}=-\frac{\partial S^{(2)}}{\partial \bar{q}_{2}}
\end{gathered}
$$

where the generating function $S^{(2)}$ for the canonical transformation (3.20) is given by

$$
\begin{gathered}
S^{(2)}=\frac{1}{4} \bar{q}_{2}<\Phi_{1}, \Phi_{1}>-\frac{1}{4} q_{1}<\bar{\Phi}_{2}, \bar{\Phi}_{2}>-\eta_{1} q_{1} \bar{p}_{1}+\eta_{1} \bar{q}_{2} p_{2}-\frac{1}{4} q_{1} \bar{q}_{2}^{2} p_{2} \\
+2 \bar{p}_{1} p_{2}+\frac{1}{4} q_{1}^{2} \bar{q}_{2} \bar{p}_{1}+\frac{1}{8} q_{1}^{2} \bar{q}_{2}^{2} \eta_{1}-\frac{1}{48} q^{3} \bar{q}_{2}^{3}+\sum_{j=1}^{N} \sqrt{\lambda_{j}-\eta_{1}} \phi_{1 j} \bar{\phi}_{2 j}-\frac{1}{3} \eta_{1}^{3} .
\end{gathered}
$$

Then it is easy to check the spectrality

$$
\begin{gathered}
\frac{\partial S^{(2)}}{\partial \eta_{1}}=\frac{1}{2} \sum_{j=1}^{N} \frac{\phi_{1 j}}{\lambda_{j}-\eta_{1}}\left[\phi_{2 j}-\frac{1}{2} \bar{q}_{2} \phi_{1 j}\right]+\bar{q}_{2} p_{2} \\
+\frac{1}{2} q_{1}\left[q_{2}-\frac{1}{4} q_{1} \bar{q}_{2}^{2}+\bar{q}_{2} \eta_{1}\right]+\frac{1}{8} q_{1}^{2} \bar{q}_{2}^{2}=A^{(2)}\left(\eta_{1}\right)+f_{1} B^{(2)}\left(\eta_{1}\right)=\mu_{1} .
\end{gathered}
$$


The point $\left(\eta_{1}, \mu_{1}\right)$ satisfies the separation equation given by the spectral curve (2.19)

$$
\mu_{1}^{2}=\eta_{1}^{4}+P_{0} \eta_{1}+P_{N+1}+\sum_{j=1}^{N} \frac{P_{j}}{\eta_{1}-\lambda_{j}} .
$$

In the exactly same way, we can find second one-point BTs for the constrained flows (2.4) according to the second DTs for the AKNS hierarchy. By composition of these two BTs, we can find two-point BTs for the constrained flows (2.4). Since these two-point BTs are quite complicate, we will present another two-point BTs for the constrained flows in the next section.

\section{Two-point BTs for high-order constrained flows of the AKNS hierarchy}

Let $\psi\left(x, \eta_{i}\right)$ be a solution of (2.7) and (2.8) with $\lambda=\eta_{i}, \mu=\mu_{i}, i=1,2, \eta_{i} \neq \lambda_{j}$. Motivated by the third DT for the AKNS hierarchy, we obtain the third DT for the constrained flows (2.4) consisting of (3.1), (3.8) and

$$
\begin{gathered}
\bar{q}=q-2 m_{1}, \quad \bar{r}=r-2 m_{3}, \\
\bar{\phi}_{1 j}=\frac{1}{\sqrt{\left(\lambda_{j}-\eta_{1}\right)\left(\lambda_{j}-\eta_{2}\right)}}\left[\left(\lambda_{j}-\eta_{1}+m_{2}\right) \phi_{1 j}-m_{1} \phi_{2 j}\right], \\
\bar{\phi}_{2 j}=\frac{1}{\sqrt{\left(\lambda_{j}-\eta_{1}\right)\left(\lambda_{j}-\eta_{2}\right)}}\left[m_{3} \phi_{1 j}+\left(\lambda_{j}-\eta_{2}-m_{2}\right) \phi_{2 j}\right] .
\end{gathered}
$$

It follows from (3.10) and (3.19)

$$
\begin{aligned}
& m_{1}=\frac{\left(\eta_{2}-\eta_{1}\right) B^{(n)}\left(\eta_{1}\right) B^{(n)}\left(\eta_{2}\right)}{\left(\mu_{2}-A^{(n)}\left(\eta_{2}\right)\right) B^{(n)}\left(\eta_{1}\right)-\left(\mu_{1}-A^{(n)}\left(\eta_{1}\right)\right) B^{(n)}\left(\eta_{2}\right)}, \\
& m_{3}=\frac{\left(\eta_{2}-\eta_{1}\right)\left(\mu_{1}-A^{(n)}\left(\eta_{1}\right)\right)\left(\mu_{2}-A^{(n)}\left(\eta_{2}\right)\right)}{\left(\mu_{2}-A^{(n)}\left(\eta_{2}\right)\right) B^{(n)}\left(\eta_{1}\right)-\left(\mu_{1}-A^{(n)}\left(\eta_{1}\right)\right) B^{(n)}\left(\eta_{2}\right)}, \\
& m_{2}=\frac{\left(\eta_{2}-\eta_{1}\right)\left(\mu_{1}-A^{(n)}\left(\eta_{1}\right)\right) B^{(n)}\left(\eta_{2}\right)}{\left(\mu_{2}-A^{(n)}\left(\eta_{2}\right)\right) B^{(n)}\left(\eta_{1}\right)-\left(\mu_{1}-A^{(n)}\left(\eta_{1}\right)\right) B^{(n)}\left(\eta_{2}\right)} .
\end{aligned}
$$


By substituting (4.2), (4.1) gives rise to infinite number $(n=0,1, \ldots)$ of explicit twopoint BT for the constrained flows (2.4). We now show that the two-point BTs (4.1) are canonical transformations and possess the spectrality property. It is easy to check that

$$
\begin{gathered}
m_{2}^{2}+\left(\eta_{2}-\eta_{1}\right) m_{2}-m_{1} m_{3}=0 \\
f_{1}=\frac{m_{2}}{m_{1}}, \quad f_{2}=\frac{m_{2}+\eta_{2}-\eta_{1}}{m_{1}} .
\end{gathered}
$$

Using (4.3), (4.1b) and (4.10) can be rewritten as

$$
\begin{gathered}
\phi_{2 j}=\frac{1}{m_{1}}\left[\left(\lambda_{j}-\eta_{1}+m_{2}\right) \phi_{1 j}-\sqrt{\left(\lambda_{j}-\eta_{1}\right)\left(\lambda_{j}-\eta_{2}\right)} \bar{\phi}_{1 j}\right], \\
\bar{\phi}_{2 j}=\frac{1}{m_{1}}\left[\sqrt{\left(\lambda_{j}-\eta_{1}\right)\left(\lambda_{j}-\eta_{2}\right)} \phi_{1 j}+\left(-\lambda_{j}+\eta_{2}+m_{2}\right) \bar{\phi}_{1 j}\right] .
\end{gathered}
$$

The formulas (3.8), (3.15) and (4.3) lead to

$$
\begin{gathered}
{\left[\lambda^{2}-\lambda\left(\eta_{1}+\eta_{2}\right)+\eta_{1} \eta_{2}\right] \bar{A}^{(n)}(\lambda)=\left[\lambda^{2}-\lambda\left(\eta_{1}+\eta_{2}\right)+\eta_{1} \eta_{2}-2 m_{1} m_{3}\right] A^{(n)}(\lambda)} \\
-m_{3}\left[\lambda-\eta_{1}+m_{2}\right] B^{(n)}(\lambda)-m_{1}\left[\lambda-\eta_{2}-m_{2}\right] C^{(n)}(\lambda), \\
{\left[\lambda^{2}-\lambda\left(\eta_{1}+\eta_{2}\right)+\eta_{1} \eta_{2}\right] \bar{B}^{(n)}(\lambda)=2 m_{1}\left[\lambda-\eta_{1}+m_{2}\right] A^{(n)}(\lambda)} \\
+\left[\lambda-\eta_{1}+m_{2}\right]^{2} B^{(n)}(\lambda)-m_{1}^{2} C^{(n)}(\lambda), \\
{\left[\lambda^{2}-\lambda\left(\eta_{1}+\eta_{2}\right)+\eta_{1} \eta_{2}\right] \bar{C}^{(n)}(\lambda)=2 m_{3}\left[\lambda-\eta_{2}-m_{2}\right] A^{(n)}(\lambda)} \\
-m_{3}^{2} B^{(n)}(\lambda)+\left[\lambda-\eta_{2}-m_{2}\right]^{2} C^{(n)}(\lambda) .
\end{gathered}
$$

(1) For the first constrained flow, the FDIHS (2.11), using (4.3) and (4.5), one gets

$$
\begin{gathered}
m_{1}=\frac{1}{4}<\bar{\Phi}_{1}, \bar{\Phi}_{1}>-\frac{1}{4}<\Phi_{1}, \Phi_{1}> \\
m_{2}=\frac{1}{<\bar{\Phi}_{1}, \bar{\Phi}_{1}>-<\Phi_{1}, \Phi_{1}>}\left[<\Lambda \bar{\Phi}_{1}, \bar{\Phi}_{1}>+<\Lambda \Phi_{1}, \Phi_{1}>\right. \\
\left.-\eta_{2}<\bar{\Phi}_{1}, \bar{\Phi}_{1}>-\eta_{1}<\Phi_{1}, \Phi_{1}>-2 \sum_{j=1}^{N} \sqrt{\left(\lambda_{j}-\eta_{1}\right)\left(\lambda_{j}-\eta_{2}\right)} \phi_{1 j} \bar{\phi}_{1 j}\right] .
\end{gathered}
$$


Then substituting (4.6) into (4.4) gives rise to

$$
\phi_{2 j}=\frac{\partial S^{(0)}}{\partial \phi_{1 j}}, \quad \bar{\phi}_{2 j}=-\frac{\partial S^{(0)}}{\partial \bar{\phi}_{1 j}}, \quad j-1, \ldots, N,
$$

where the generating function $S^{(0)}$ for the canonical transformation (4.1) is given by

$$
\begin{gathered}
S^{(0)}=\frac{2}{<\bar{\Phi}_{1}, \bar{\Phi}_{1}>-<\Phi_{1}, \Phi_{1}>}\left[<\Lambda \bar{\Phi}_{1}, \bar{\Phi}_{1}>+<\Lambda \Phi_{1}, \Phi_{1}>-\eta_{2}<\bar{\Phi}_{1}, \bar{\Phi}_{1}>\right. \\
\left.-\eta_{1}<\Phi_{1}, \Phi_{1}>-2 \sum_{j=1}^{N} \sqrt{\left(\lambda_{j}-\eta_{1}\right)\left(\lambda_{j}-\eta_{2}\right)} \phi_{1 j} \bar{\phi}_{1 j}\right]-\eta_{1}-\eta_{2} .
\end{gathered}
$$

Furthermore, it is found

$$
\begin{gathered}
\frac{\partial S^{(0)}}{\partial \eta_{1}}=-1+\frac{2}{<\bar{\Phi}_{1}, \bar{\Phi}_{1}>-<\Phi_{1}, \Phi_{1}>}\left[-<\Phi_{1}, \Phi_{1}>+2 \sum_{j=1}^{N} \sqrt{\frac{\left(\lambda_{j}-\eta_{2}\right)}{\left(\lambda_{j}-\eta_{1}\right)}} \phi_{1 j} \bar{\phi}_{1 j}\right] \\
=A^{(0)}\left(\eta_{1}\right)+f_{1} B^{(0)}\left(\eta_{1}\right)=\mu_{1} \\
\frac{\partial S^{(0)}}{\partial \eta_{2}}=A^{(0)}\left(\eta_{2}\right)+f_{2} B^{(0)}\left(\eta_{2}\right)=\mu_{2} .
\end{gathered}
$$

This implies that $\left(\eta_{1}, \mu_{1}\right)$ and $\left(\eta_{2}, \mu_{2}\right)$ satisfy the spectrality property and the separation equations given by the spectral curve (2.13)

$$
\mu_{i}^{2}=1+\sum_{j=1}^{N} \frac{P_{j}}{\eta_{i}-\lambda_{j}}, \quad i=1,2
$$

(2) For the second constrained flow, the FDIHS (2.14), formula (4.5) gives rise to

$$
\begin{gathered}
m_{1}=\frac{1}{2}(q-\bar{q}), \\
m_{2}=\frac{1}{q+\bar{q}}\left[\frac{1}{4}<\bar{\Phi}_{1}, \bar{\Phi}_{1}>-\frac{1}{4}<\Phi_{1}, \Phi_{1}>+\eta_{1} q-\eta_{2} \bar{q}\right],
\end{gathered}
$$

and

$$
r=\frac{4}{(q-\bar{q})^{2}}\left[-\frac{1}{4}<\Lambda \bar{\Phi}_{1}, \bar{\Phi}_{1}>-\frac{1}{4}<\Lambda \Phi_{1}, \Phi_{1}>+\frac{1}{4}\left(\eta_{1}+\eta_{2}\right)<\bar{\Phi}_{1}, \bar{\Phi}_{1}>\right.
$$




$$
\begin{gathered}
\left.-\eta_{1} \eta_{2} \bar{q}+\left(m_{2}-\eta_{1}\right)^{2} q+\frac{1}{2} \sum_{j=1}^{N} \sqrt{\left(\lambda_{j}-\eta_{1}\right)\left(\lambda_{j}-\eta_{2}\right)} \phi_{1 j} \bar{\phi}_{1 j}\right], \\
\bar{r}=-2 \frac{m_{2}}{m_{1}}\left(m_{2}+\eta_{2}-\eta_{1}\right)+r .
\end{gathered}
$$

By substitution of (4.10), 4.4) and (4.11) can be rewritten as

$$
\phi_{2 j}=\frac{\partial S^{(1)}}{\partial \phi_{1 j}}, \quad \bar{\phi}_{2 j}=-\frac{\partial S^{(1)}}{\partial \bar{\phi}_{1 j}}, \quad r=\frac{\partial S^{(1)}}{\partial q}, \quad \bar{r}=-\frac{\partial S^{(1)}}{\partial \bar{q}},
$$

where the generating function $S^{(1)}$ for the canonical transformation (4.1) is given by

$$
\begin{gathered}
S^{(1)}=\frac{1}{q-\bar{q}}\left[<\Lambda \bar{\Phi}_{1}, \bar{\Phi}_{1}>+<\Lambda \Phi_{1}, \Phi_{1}>-2 \sum_{j=1}^{N} \sqrt{\left(\lambda_{j}-\eta_{1}\right)\left(\lambda_{j}-\eta_{2}\right)} \phi_{1 j} \bar{\phi}_{1 j}\right] \\
+\frac{1}{q^{2}-\bar{q}^{2}}\left[\frac{1}{4}<\bar{\Phi}_{1}, \bar{\Phi}_{1}><\Phi_{1}, \Phi_{1}>-\left(\eta_{1}+\eta_{2}\right) q<\bar{\Phi}_{1}, \bar{\Phi}_{1}>-\left(\eta_{1}+\eta_{2}\right) \bar{q}<\Phi_{1}, \Phi_{1}>\right. \\
\left.-\frac{1}{8}<\bar{\Phi}_{1}, \bar{\Phi}_{1}>^{2}-\frac{1}{8}<\Phi_{1}, \Phi_{1}>^{2}+4 \eta_{1} \eta_{2} q \bar{q}-2\left(\eta_{1}^{2}+\eta_{2}^{2}\right) \bar{q}^{2}\right]-\eta_{1}^{2}-\eta_{2}^{2} .
\end{gathered}
$$

It is easy to check the spectrality property

$$
\begin{gathered}
\frac{\partial S^{(1)}}{\partial \eta_{1}}=\frac{1}{q^{2}-\bar{q}^{2}}\left[-q<\bar{\Phi}_{1}, \bar{\Phi}_{1}>-\bar{q}<\Phi_{1}, \Phi_{1}>-4 \eta_{1} \bar{q}^{2}+4 \eta_{2} q \bar{q}\right]-2 \eta_{1} \\
+\frac{1}{q-\bar{q}} \sum_{j=1}^{N} \sqrt{\frac{\lambda_{j}-\eta_{2}}{\lambda_{j}-\eta_{1}}} \phi_{1 j} \bar{\phi}_{1 j}=-2\left[A^{(1)}\left(\eta_{1}\right)+f_{1} B^{(1)}\left(\eta_{1}\right)\right]=-2 \mu_{1}, \\
\frac{\partial S^{(1)}}{\partial \eta_{2}}=-2\left[A^{(1)}\left(\eta_{2}\right)+f_{2} B^{(1)}\left(\eta_{2}\right)\right]=-2 \mu_{2} .
\end{gathered}
$$

The points $\left(\eta_{i}, \mu_{i}\right)$ satisfy the separation equations given by the spectral curve (2.16)

$$
\mu_{i}^{2}=\eta_{i}^{2}+P_{0}+\sum_{j=1}^{N} \frac{P_{j}}{\eta_{i}-\lambda_{j}}, \quad i=1,2 .
$$

(3) For the third constrained flow, the FDIHS (2.17), by means of (4.3) and (4.5), one gets

$$
m_{1}=\frac{1}{2}\left(q_{1}-\bar{q}_{1}\right), \quad m_{3}=\frac{1}{2}\left(q_{2}-\bar{q}_{2}\right)
$$




$$
m_{2}=\frac{1}{2}\left(\eta_{1}-\eta_{2}\right)+\frac{1}{2} \sqrt{\left(\eta_{1}-\eta_{2}\right)^{2}+\left(q_{1}-\bar{q}_{1}\right)\left(q_{2}-\bar{q}_{2}\right)}
$$

and

$$
\begin{gathered}
p_{1}=\frac{2}{\left(q_{1}-\bar{q}_{1}\right)^{2}}\left\{-\frac{1}{2}<\Lambda \Phi_{1}, \Phi_{1}>-\frac{1}{2}<\Lambda \bar{\Phi}_{1}, \bar{\Phi}_{1}>+\frac{1}{2}\left(\eta_{1}+\eta_{2}\right)<\bar{\Phi}_{1}, \bar{\Phi}_{1}>\right. \\
-m_{1}\left(m_{2}-\eta_{1}\right)^{2} q_{1} q_{2}+\left(m_{2}-\eta_{1}\right)^{2}\left(m_{2} q_{1}+m_{2} \bar{q}_{1}+\eta_{2} \bar{q}_{1}-\eta_{1} q_{1}\right) \\
+\sum_{j=1}^{N} \sqrt{\left(\lambda_{j}-\eta_{1}\right)\left(\lambda_{j}-\eta_{2}\right)} \phi_{1 j} \bar{\phi}_{1 j} \\
+\frac{\eta_{1} \eta_{2}-\left(m_{2}-\eta_{1}\right)^{2}}{\sqrt{\left(\eta_{1}-\eta_{2}\right)^{2}+\left(q_{1}-\bar{q}_{1}\right)\left(q_{2}-\bar{q}_{2}\right)}}\left[-\frac{1}{2}<\bar{\Phi}_{1}, \bar{\Phi}_{1}>+\frac{1}{2}<\Phi_{1}, \Phi_{1}>\right. \\
\left.\left.-\frac{1}{4} q_{2}\left(q_{1}^{2}-\bar{q}_{1}^{2}\right)+\left(m_{2}-\eta_{1}\right)^{2}\left(q_{1}+2 \bar{q}_{1}\right)+2\left(m_{2}-\eta_{1}\right)\left(\eta_{1}+\eta_{2}\right) \bar{q}_{1}+\eta_{1} \eta_{2} \bar{q}_{1}\right]\right\} \\
\bar{p}_{1}=p_{1}+\frac{1}{2}\left(m_{2}+\eta_{2}\right)\left(q_{2}+\bar{q}_{2}\right)-\frac{1}{2}\left(\eta_{1}+\eta_{2}\right) \bar{q}_{2}, \\
\left.\frac{1}{2}=\frac{1}{4} q_{2}\left(q_{1}^{2}-\bar{q}_{1}^{2}\right)+\left(m_{2}-\eta_{1}\right)^{2}\left(q_{1}+2 \bar{q}_{1}\right)+2\left(m_{2}-\eta_{1}\right)\left(\eta_{1}+\eta_{2}\right) \bar{q}_{1}+\eta_{1} \eta_{2} \bar{q}_{1}\right], \\
\frac{p_{2}=\bar{p}_{2}+\frac{1}{2}\left(\eta_{1}+\eta_{2}\right) q_{1}-\frac{1}{2}}{2}\left(m_{2}+\eta_{2}\right)\left(q_{1}+\bar{q}_{1}\right) .
\end{gathered}
$$

By inserting (4.15) into (4.4) and (4.16), a straightforward calculation leads to

$$
\begin{array}{ll}
\phi_{2 j}=\frac{\partial S^{(2)}}{\partial \phi_{1 j}}, & \bar{\phi}_{2 j}=-\frac{\partial S^{(2)}}{\partial \bar{\phi}_{1 j}}, \\
p_{2}=\frac{\partial S^{(2)}}{\partial q_{2}}, & p_{1}=\frac{\partial S^{(2)}}{\partial q_{1}} \\
\bar{p}_{1}=-\frac{\partial S^{(2)}}{\partial \bar{q}_{1}}, & \bar{p}_{2}=-\frac{\partial S^{(2)}}{\partial \bar{q}_{2}}
\end{array}
$$

where the generating function $S^{(2)}$ for the canonical transformation (4.1) is given by

$$
\begin{aligned}
S^{(2)}= & \frac{1}{q_{1}-\bar{q}_{1}}\left[<\Lambda \bar{\Phi}_{1}, \bar{\Phi}_{1}>+<\Lambda \Phi_{1}, \Phi_{1}>-\frac{1}{2}\left(\eta_{1}+\eta_{2}\right)\left(<\bar{\Phi}_{1}, \bar{\Phi}_{1}>+<\Phi_{1}, \Phi_{1}>\right)\right. \\
& +\frac{2}{3}\left(m_{2}-\eta_{1}\right)^{3}\left(q_{1}+2 \bar{q}_{1}\right)+2\left(m_{2}-\eta_{1}\right)^{2}\left(\eta_{1}+\eta_{2}\right) \bar{q}_{1}-\left(\eta_{1}+\eta_{2}\right) \eta_{1} \eta_{2} \bar{q}_{1}
\end{aligned}
$$




$$
\begin{gathered}
+\frac{1}{2} \sqrt{\left(\eta_{1}-\eta_{2}\right)^{2}+\left(q_{1}-\bar{q}_{1}\right)\left(q_{2}-\bar{q}_{2}\right)}\left(<\Phi_{1}, \Phi_{1}>-<\bar{\Phi}_{1}, \bar{\Phi}_{1}>+2 \eta_{1} \eta_{2} \bar{q}_{1}\right) \\
\left.-2 \sum_{j=1}^{N} \sqrt{\left(\lambda_{j}-\eta_{1}\right)\left(\lambda_{j}-\eta_{2}\right)} \phi_{1 j} \bar{\phi}_{1 j}\right]+\frac{1}{4}\left(\eta_{1}+\eta_{2}\right)\left(q_{1}-\bar{q}_{1}\right) q_{2} \\
-\frac{1}{4} \sqrt{\left(\eta_{1}-\eta_{2}\right)^{2}+\left(q_{1}-\bar{q}_{1}\right)\left(q_{2}-\bar{q}_{2}\right)} q_{2}\left(q_{1}+\bar{q}_{1}\right)+\frac{1}{3}\left(\eta_{1}^{3}+\eta_{2}^{3}\right) .
\end{gathered}
$$

By a straightforward calculation, we can show the spectrality property

$$
\frac{\partial S^{(2)}}{\partial \eta_{1}}=A^{(2)}\left(\eta_{1}\right)+f_{1} B^{(2)}\left(\eta_{1}\right)=\mu_{1}, \quad \frac{\partial S^{(2)}}{\partial \eta_{2}}=A^{(2)}\left(\eta_{2}\right)+f_{2} B^{(2)}\left(\eta_{2}\right)=\mu_{2} .
$$

The points $\left(\eta_{i}, \mu_{i}\right)$ satisfy the separation equations given by the spectral curve $(2.19)$

$$
\mu_{i}^{2}=\eta_{i}^{4}+P_{0} \eta_{i}+P_{N+1}+\sum_{j=1}^{N} \frac{P_{j}}{\eta_{i}-\lambda_{j}}, \quad i=1,2 .
$$

\section{5 m-times repeated two-point DTs for high-order con- strained flows of the AKNS hierarchy}

Assume that $\left(\psi_{1}\left(x, \eta_{i}\right), \psi_{2}\left(x, \eta_{i}\right)\right)^{T}, i=1, \ldots, 2 m$, be solutions of (2.7) and (2.8) with $\lambda=\eta_{i}, \mu=\mu_{i}, i=1,2, \ldots, 2 m, \eta_{i} \neq \lambda_{j}$. We use $q[l], r[l], \phi_{1 j}[l], \phi_{2 j}[l]$ to denote the action of l-times repeated two-point DTs of (4.1) on the initial solution $q[0], r[0], \phi_{1 j}[0], \phi_{2 j}[0]$. We have according to $(4.1)$

$$
\begin{gathered}
q[l+1]=q[l]-2 m_{1}[l], \quad r[l+1]=r[l]-2 m_{3}[l], \\
\phi_{1 j}[l+1]=\frac{1}{\sqrt{\left(\lambda_{j}-\eta_{2 l+1}\right)\left(\lambda_{j}-\eta_{2 l+2}\right)}}\left[\left(\lambda_{j}-\eta_{2 l+1}+m_{2}[l]\right) \phi_{1 j}[l]-m_{1}[l] \phi_{2 j}[l]\right], \\
\phi_{2 j}[l+1]=\frac{1}{\sqrt{\left(\lambda_{j}-\eta_{2 l+1}\right)\left(\lambda_{j}-\eta_{2 l+2}\right)}}\left[m_{3}[l] \phi_{1 j}[l]+\left(\lambda_{j}-\eta_{2 l+2}-m_{2}[l]\right) \phi_{2 j}[l]\right] .
\end{gathered}
$$

We denote

$$
G_{m}=\left(\begin{array}{ccccc}
\eta_{1}^{m} \psi_{1}\left(\eta_{1}\right) & \eta_{2}^{m} \psi_{1}\left(\eta_{2}\right) & \eta_{3}^{m} \psi_{1}\left(\eta_{3}\right) & \ldots \ldots & \eta_{2 m}^{m} \psi_{1}\left(\eta_{2 m}\right) \\
\eta_{1}^{m-1} \psi_{1}\left(\eta_{1}\right) & \eta_{2}^{m-1} \psi_{1}\left(\eta_{2}\right) & \eta_{3}^{m-1} \psi_{1}\left(\eta_{3}\right) & \ldots \ldots & \eta_{2 m}^{m-1} \psi_{1}\left(\eta_{2 m}\right) \\
\ldots \ldots & \ldots \ldots & \ldots \ldots & \ldots \ldots & \ldots \ldots \\
\psi_{1}\left(\eta_{1}\right) & \psi_{1}\left(\eta_{2}\right) & \psi_{1}\left(\eta_{3}\right) & \ldots \ldots & \psi_{1}\left(\eta_{2 m}\right) \\
\eta_{1}^{m-2} \psi_{2}\left(\eta_{1}\right) & \eta_{2}^{m-2} \psi_{2}\left(\eta_{2}\right) & \eta_{3}^{m-2} \psi_{2}\left(\eta_{3}\right) & \ldots \ldots & \eta_{2 m}^{m-2} \psi_{2}\left(\eta_{2 m}\right) \\
\ldots \ldots & \ldots \ldots & \ldots \ldots & \ldots \ldots & \ldots \ldots \\
\psi_{2}\left(\eta_{1}\right) & \psi_{2}\left(\eta_{2}\right) & \psi_{2}\left(\eta_{3}\right) & \ldots \ldots & \psi_{2}\left(\eta_{2 m}\right)
\end{array}\right)
$$




$$
\begin{gathered}
\triangle_{m}=\left(\begin{array}{ccccc}
\eta_{1}^{m-1} \psi_{1}\left(\eta_{1}\right) & \eta_{2}^{m-1} \psi_{1}\left(\eta_{2}\right) & \eta_{3}^{m-1} \psi_{1}\left(\eta_{3}\right) & \ldots \ldots & \eta_{2 m}^{m-1} \psi_{1}\left(\eta_{2 m}\right) \\
\eta_{1}^{m-2} \psi_{1}\left(\eta_{1}\right) & \eta_{2}^{m-2} \psi_{1}\left(\eta_{2}\right) & \eta_{3}^{m-2} \psi_{1}\left(\eta_{3}\right) & \ldots \ldots & \eta_{2 m}^{m-2} \psi_{1}\left(\eta_{2 m}\right) \\
\ldots \ldots & \ldots \ldots & \ldots \ldots & \ldots \ldots & \ldots \ldots \\
\psi_{1}\left(\eta_{1}\right) & \psi_{1}\left(\eta_{2}\right) & \psi_{1}\left(\eta_{3}\right) & \ldots \ldots & \psi_{1}\left(\eta_{2 m}\right) \\
\eta_{1}^{m-1} \psi_{2}\left(\eta_{1}\right) & \eta_{2}^{m-1} \psi_{2}\left(\eta_{2}\right) & \eta_{3}^{m-1} \psi_{2}\left(\eta_{3}\right) & \ldots \ldots & \eta_{2 m}^{m-1} \psi_{2}\left(\eta_{2 m}\right) \\
\ldots \ldots & \ldots \ldots & \ldots \ldots & \ldots \ldots & \ldots \ldots \\
\psi_{2}\left(\eta_{1}\right) & \psi_{2}\left(\eta_{2}\right) & \psi_{2}\left(\eta_{3}\right) & \ldots \ldots & \psi_{2}\left(\eta_{2 m}\right)
\end{array}\right) \\
W_{m}(j)=\left(\begin{array}{ccccc}
\lambda_{j}^{m} \phi_{1 j}[0] & \eta_{1}^{m} \psi_{1}\left(\eta_{1}\right) & \eta_{2}^{m} \psi_{1}\left(\eta_{2}\right) & \ldots \ldots & \eta_{2 m}^{m} \psi_{1}\left(\eta_{2 m}\right) \\
\lambda_{j}^{m-1} \phi_{1 j}[0] & \eta_{1}^{m-1} \psi_{1}\left(\eta_{1}\right) & \eta_{2}^{m-1} \psi_{1}\left(\eta_{2}\right) & \ldots \ldots & \eta_{2 m}^{m-1} \psi_{1}\left(\eta_{2 m}\right) \\
\ldots \ldots & \ldots \ldots & \ldots \ldots & \ldots \ldots & \ldots \ldots \\
\phi_{1 j}[0] & \psi_{1}\left(\eta_{1}\right) & \psi_{1}\left(\eta_{2}\right) & \ldots \ldots & \psi_{1}\left(\eta_{2 m}\right) \\
\lambda_{j}^{m-1} \phi_{2 j}[0] & \eta_{1}^{m-1} \psi_{2}\left(\eta_{1}\right) & \eta_{2}^{m-1} \psi_{2}\left(\eta_{2}\right) & \ldots \ldots & \eta_{2 m}^{m-1} \psi_{2}\left(\eta_{2 m}\right) \\
\ldots \ldots & \ldots \ldots & \ldots \ldots & \ldots \ldots & \ldots \ldots \\
\phi_{2 j}[0] & \psi_{2}\left(\eta_{1}\right) & \psi_{2}\left(\eta_{2}\right) & \ldots \ldots & \psi_{2}\left(\eta_{2 m}\right)
\end{array}\right) .
\end{gathered}
$$

Then $m$-times repeated DTs for the constrained flows (2.4) are given by

$$
\begin{gathered}
q[m]=q[0]-2 \frac{G_{m}}{\triangle_{m}^{*}}, \quad r[m]=r[0]-2 \frac{G_{m}^{*}}{\triangle_{m}^{*}}, \\
\phi_{1 j}[m]=\frac{1}{\sqrt{\prod_{i=1}^{2 m}\left(\lambda_{j}-\eta_{i}\right)}} \frac{W_{m}(j)}{\triangle_{m}}, \quad \phi_{2 j}[m]=\frac{1}{\sqrt{\prod_{i=1}^{2 m}\left(\lambda_{j}-\eta_{i}\right)}} \frac{W_{m}^{*}(j)}{\triangle_{m}^{*}},
\end{gathered}
$$

where $G_{m}^{*}, W_{m}^{*}(j)$ and $\triangle_{m}^{*}$ are obtained by interchanging $\psi_{1}\left(x, \eta_{i}\right)$ and $\psi_{2}\left(x, \eta_{i}\right), i=$ $1, \ldots, 2 m, \phi_{1 j}\left(\lambda_{j}\right)$ and $\phi_{2 j}\left(\lambda_{j}\right)$ in $G_{m}, W_{m}(j)$ and $\triangle_{M}$, respectively. Formula (5.2a) was shown in [2, 16], (5.2b) can be obtained in the same way by using the formulas for Vandermonde-like determinants $G_{m}^{*}, W_{m}^{*}(j)$ and $\triangle_{m}^{*}$ given in [22].

\section{Conclusion}

Some methods were presented to construct the Bäcklund transformations with the properties described above in [6, 7, 8, 9, 10] for few examples. In this paper we propose a way to construct infinite number of explicit one- and two-point Bäcklund transformations for high-order constrained flows of soliton hierarchy by means of the Darboux transformations for the constrained flows by using the high-order constrained flows of the AKNS 
hierarchy as model. By constructing the generating functions, it is shown that these BTs are canonical transformations including Bäcklund parameter $\eta$ and a spectrality property holds with respect to the Bäcklund parameter $\eta$ and the conjugate variable $\mu$. The pair $(\eta, \mu)$ lies on the spectral curve and satisfies the separation equation. Also we present the formula for m-times repeated Darboux transformations for the high-order constrained flows of the AKNS hierarchy.

The method prososed in this paper can be applied to the high-order binary constrained flows of AKNS hierarchy in [23] to find new explicit BT's with canonicity and spectrality.

\section{Acknowledgment}

The work described in this paper was supported by a grant from CityU (project No. 7001072) and the Special Funds for Chinese Major Basic Research Project "Nonlinear Science".

\section{References}

[1] Rogers C and Sahdwick W F 1982 Bäcklund transformations and their applications (New York: Academic Press).

[2] Matveev V B and Salle M A 1991 Darboux Transformations and Solitons (Berlin: Springer)

[3] Flaschka H and Laughlin D Mc 1976 in Bäcklund Transformations, ed. by Miura R, Lect. Notes Math. Vol 515 (Berling Heideberg: Springer) 225.

[4] Toda M and Wadati M 1975 J. Phys. Soc. Japan 39, 1204.

[5] Kodama Y and Wadati M 1976 Progr. Thoer. Phys. 56, 342 and 1740.

[6] Kuznetsov V B and Sklyanin E K 1998 J. Phys. A 31, 2241.

[7] Kuznetsov V B and Vanhaecke P 2000 Bäcklund transformation for finitedimensional integrable system: a geometric approach, to appear in Geometry and Physics.

[8] Sklyanin E K 2000 Amer. Math. Soc. Transt. Ser. 2, 201. 
[9] Hone A N W, Kuznetsov V B and Ragnisco O 1999 J. Phys. A: Math. Gen. 32, L299.

[10] Hone A N W, Kuznetsov V B and Ragnisco O 2001 J. Phys. A: Math. Gen. 34, 2477.

[11] Zeng Yunbo 1991 Phys. Lett. A 160, 541.

[12] Ragnisco O and Rauch-Wojciechowski S 1992 Inverse Problems 8, 245.

[13] Ma W X, Strampp W 1994 Phys. Lett. A 185, 277.

[14] Zeng Yunbo and Li Yishen 1993 J. Phys. A 26, L273.

[15] Zeng Yunbo 1994 Physica D 73, 171.

[16] Its A R, Salle M A and Rybin A V 1988 Teor. Mat. Fiz 74(1), 29.

[17] Gu Chaohao and Zhou Zixing 1987 Lett. Math. Phys. 12, 169.

[18] Li Yishen, Gu Xinshen and Zhou Maorong 1987 Acta Math. Sinica 3, 143.

[19] Zeng Yunbo 1995 Acta Math. Scientia 15, 337.

[20] Zeng Yunbo and Li Yishen 1996 Acta Math. Sinica, New Series, 12, 217.

[21] Ablowitz M J and Segur H 1981 Solitons and the Inverse Scattering Transform ( Philadelphia: SIAM)

[22] Steudel H, Meinel R and Neugebauer G 1997 J. Math. Phys. 38, 4692.

[23] Li Yishen and Ma Wenxiu 2000 Chaos, Soliton and Fraclas 11, 697. 